\title{
Implementación y evaluación de un programa de inteligencia emocional en adolescentes*
}

\section{Implementation and Evaluation of an Emotional Intelligence Program in Adolescents}

\author{
Víctor Santiago Sigüenza-Marín ${ }^{\mathrm{a}}$ \\ Universidad de La Rioja, España \\ Raguel Carballido-Guisado \\ Universidad de La Rioja, España \\ Alicia Pérez-Albéniz \\ Universidad de La Rioja, España \\ ORCID: http://orcid.org/0000-0002-7182-060X \\ Eduardo Fonseca-Pedrero \\ Universidad de La Rioja, España \\ ORCID: https://orcid.org/0000-0001-7453-5225
}

a Autor de correspondencia. Correo electrónico: eduardo.fonseca@unirioja.es

Para citar este artículo: Sigüenza-Marín, V. S., CarballidoGuisado, R., Pérez-Albéniz, A., \& Fonseca-Pedrero, E. (2019). Implementación y evaluación de un programa de inteligencia emocional en adolescentes. Universitas Psychologica, 18(3), 1-13. https://doi.org/10.11144/ Javeriana.upsy18-3.iepi

\section{RESUMEN}

El principal objetivo de este trabajo fue implementar y evaluar un programa de inteligencia emocional en adolescentes. Se utilizaron un grupo experimental $(n=60)$ y un grupo de comparación $(n=44)$ que fueron evaluados en dos momentos temporales con el Trait Meta-Mood Scale-24, el Cuestionario de Capacidades y Dificultades, el Índice de Reactividad Interpersonal y la Escala de Autoestima de Rosenberg. Los resultados mostraron que, tras un periodo de tres meses de intervención, el grupo experimental no difirió entre el pretest y el posttest de forma estadísticamente significativa en las puntuaciones medias de inteligencia emocional percibida, empatía, salud mental o autoestima. La satisfacción del programa informada por los alumnos fue excelente. A pesar de los resultados obtenidos, es necesario seguir potencializando las habilidades emocionales y sociales en esta etapa del desarrollo con la finalidad de mejorar el ajuste personal y la calidad de vida, así como el desarrollo integral de los adolescentes.

\section{Palabras clave}

adolescentes; inteligencia emocional; educación emocional; intervención; evaluación.

\begin{abstract}
The main aim was to implement and evaluate an emotional intelligence intervention in an educational setting. An experimental group $(n=60)$ and a control group $(n=44)$ were evaluated in two different moments with the Trait Meta-Mood Scale-24, the Strengths and Difficulties Questionnaire, the Interpersonal Reactivity Index, and the Rosenberg's Self-Esteem Scale. The results showed that, after three months of intervention, no statistically significant differences were found between pre-test and post-test in the median scoring of emotional intelligence, empathy, mental health, and self-esteem. The satisfaction of the program
\end{abstract}


reported by the students was excellent. Despite the results obtained, it is necessary to continue strengthening the social-emotional skills in this stage of development to improve the personal adjustment and quality of life, as well as the comprehensive development of the adolescents.

Keywords

adolescents; emotional intelligence; emotional education; intervention; assessment.

Las dificultades relacionadas con el estrés, la ansiedad, la depresión, la ideación suicida, los comportamientos disruptivos, entre otros, se encuentran entre los principales problemas de salud de los jóvenes (Bousoño et al., 2017; Carli et al., 2014; Merikangas et al., 2010; Sánchez, Pérez, Paino, y Fonseca-Pedrero, 2018). El impacto y las consecuencias negativas a nivel personal, familiar, educativo, social y sanitario asociadas a estos problemas de salud mental son evidentes (Catalá-López, Gènova-Maleras, Álvarez-Martín, de Larrea-Baz, y Morant-Ginestar, 2013; Parés-Badell et al., 2014). Además, estudios previos indican que aquellas personas que saben cómo gestionar y regular sus emociones tienen mejores resultados a nivel educativo y laboral, así como menos problemas de salud mental, mayor calidad de vida y satisfacción personal (e.g., Bisquerra, PérezGonzález, \& García, 2015; Cabello, Castillo, Rueda, \& Fernández-Berrocal, 2016; MestreNavas y Fernández-Berrocal, 2007; Ruiz-Aranda et al., 2012; Ruiz-Aranda, Salguero, Cabello, Palomera, \& Fernández-Berrocal, 2011). Es por ello que en los últimos años han surgido diferentes acercamientos, tanto en el ámbito educativo como asistencial, que tienen como base educar los aspectos emocionales, esto es, desarrollar y mejorar las competencias socioemocionales de los jóvenes como forma de promoción de la salud, prevención de trastornos psicológicos y desarrollo integral del alumno. Este conjunto de actuaciones se recoge bajo diferentes etiquetas como, por ejemplo, programas de educación emocional, promoción del bienestar emocional o de resiliencia y empoderamiento, por citar algunos.

La educación emocional es un procedimiento didáctico, prolongado y estable, que pretende aumentar al máximo el potencial emocional como sustento esencial para el desarrollo cognitivo, estableciendo ambos componentes como imprescindibles para el desarrollo de la personalidad integral (Bisquerra, 2011; Bisquerra et al., 2015). Existen muchos modelos o formas de conceptualizar la Inteligencia Emocional (IE) (Bisquerra et al., 2015; ExtremeraPacheco \& Fernández-Berrocal, 2013). Entre los acercamientos teóricos con mayor sustento científico se encuentra el modelo de habilidad de Mayer y Salovey (1997). Se conceptualiza la IE como un conjunto de cuatro habilidades referidas a a) percibir, valorar y expresar emociones con exactitud; b) acceder y/o generar sentimientos que faciliten el pensamiento; d) comprender emociones y el conocimiento emocional, y e) regular las emociones promoviendo un crecimiento emocional e intelectual. Según estos autores, la IE se puede ver como una forma de unificar y adaptar las emociones a nuestro pensamiento, indistintamente de las peculiaridades permanentes de personalidad. Estos autores no incluyen aspectos más próximos a la personalidad, justificando innecesario volver a clasificar todas las partes de la personalidad como IE (Mayer, Caruso, \& Salovey, 2000; Mayer, Roberts, \& Barsade, 2008). Para considerar que una persona es emocionalmente inteligente se debe desarrollar y enlazar las cuatro ramas que componen la IE, debido a que el óptimo desarrollo de una de ellas no asegura una IE completa. Por ello, resulta necesario dominar cada uno de sus componentes. Esto se puede ver reflejado en personas que son muy habilidosas para percibir y expresar sus emociones, pero son incapaces de regularlas y comprenderlas (Fernández-Berrocal \& Extremera-Pacheco, 2002).

Son numerosos los estudios que demuestran los beneficios en los jóvenes adolescentes cuando se aumentan sus habilidades emocionales (Castillo, Salguero, Fernández-Berrocal, \& Balluerka, 2013; Ruiz-Aranda et al., 2012; Extremera-Pacheco \& Fernández-Berrocal, 2004; Fernández-Berrocal et al., 2004). Estos resultados han impulsado, entre otros aspectos, diversos programas de desarrollo y mejora de las 
competencias de tinte socio-emocional tanto a nivel nacional como internacional. Por ejemplo, en EEUU se ha desarrollado el programa Social Emotional Learning (SEL), iniciado por Goleman en 1995, con la finalidad de alcanzar no solo el éxito en la escuela sino también en la vida cotidiana. En el ámbito nacional se encuentran propuestas como el Modelo GROP (Grop de Recerca en Orientació Psicopedagógica) el cual añade competencias emocionales diferentes en función de cinco grandes bloques (Bisquerra et al., 2015). Asimismo, la Fundación Botín establece un programa denominado Educación Responsable con el fin de mejorar el equilibrio y bienestar de los jóvenes (Repetto-Talavera \& Pérez-González, 2007). También, promovido por el equipo de Fernández Berrocal, se encuentra el programa INTEMO (+) (Ruiz-Aranda et al., 2013; Cabello et al., 2016).

Concretamente, el programa de educación emocional INTEMO $(+)$ se compone de un conjunto de actividades dirigidos a mejorar las habilidades emocionales (Cabelloet al., 2016). Está dirigido a jóvenes de entre 12 y 18 años. Es un programa práctico en el cual la metodología que se lleva a cabo permite a los jóvenes participantes desarrollar cada una de las habilidades que componen la IE desarrollando la capacidad de percibir, expresar, facilitar, comprender y manejar las emociones propias y las de los demás, habilidades que pertenecen a las cuatro ramas del modelo teórico de Mayer y Salovey (1997). El INTEMO (+) ha sido validado experimentalmente, mediante un diseño cuasi-experimental con seguimiento longitudinal. Los resultados empíricos hallados han sido satisfactorios y avalan su utilidad y validez. Por ejemplo, ha mostrado beneficios en distintos ambientes y contextos tan importantes para los adolescentes como son la salud física y mental, las relaciones interpersonales, el rendimiento y la conducta agresiva (Castillo et al., 2013; Ruiz-Aranda, et al., 2012; RuizAranda, et al., 2011).

Es importante emplear y diseñar programas educativos de educación emocional para llevar a cabo en las aulas, cuyo propósito sea únicamente desarrollar la IE o las competencias emocionales. Es necesario, además, evaluar estas intervenciones para aportar elementos empíricos que validen y certifiquen dichos procesos y su grado de efectividad, así como ayudar a divisar los aspectos que sean convenientes de mejora (Pérez-González, 2008). El presente estudio se encuentra dentro de este contexto de investigación, teniendo como objetivo esencial la promoción del bienestar emocional y prevención de los problemas de salud mental infantojuvenil. En concreto, los objetivos de este estudio fueron a) implementar un programa de educación emocional en centros escolares con la finalidad de desarrollar, promover y/o potenciar las competencias socio-emocionales de los adolescentes; b) analizar los posibles beneficios del programa de educación emocional sobre diferentes indicadores de salud mental, autoestima, empatía e inteligencia emocional percibida; y c) analizar la satisfacción de los usuarios que han participado en el programa de intervención.

\section{Método}

\section{Diseño experimental}

El diseño de investigación fue un diseño longitudinal de tipo cuasi-experimental con evaluación pre y post tratamiento. El estudio contó con un grupo control y un grupo de tratamiento/intervención.

\section{Participantes}

La muestra estuvo compuesta por un total de 104 alumnos riojanos divida en dos grupos: un grupo experimental y un grupo control. El primer grupo experimental lo formaron 60 alumnos de Educación Secundaria Obligatoria (ESO) de un centro de ESO de La Rioja. La edad media fue 13.42 años y la desviación típica de 0.52 (rango de edad 13-15), siendo el $43.3 \%$ chicos. Los alumnos del grupo control fueron seleccionados mediante un muestreo de conveniencia, considerando las variables 
sociodemográficas referidas al género y la edad. El grupo control lo formaron 44 alumnos de la ESO seleccionados de diferentes centros escolares de la Rioja. La edad media fue 13.50 años y la desviación típica de 0.50 (rango de edad 13-15), siendo el $38.6 \%$ chicos. No se observaron diferencias estadísticamente significativas ni en función de la edad ni el género entre los grupos objeto de comparación.

\section{Instrumentos}

En la presente investigación se emplearon los siguientes instrumentos de medida:

Cuestionario de Capacidades y Dificultades (Strengths and Difficulties Questionnaire, SDQ) versión autoinformada (Goodman, 1997). El SDQ es un instrumento de medida utilizado para la evaluación de dificultades conductuales y emocionales, así como la valoración de capacidades en el ámbito social. El SDQ se compone de 25 ítems con un formato de respuesta Likert de tres opciones $(0=\mathrm{No}$, nunca, $1=\mathrm{A}$ veces, $2=$ Sí, siempre). Los ítems se agrupan en cinco subescalas (con cinco ítems cada una): Dificultades Emocionales, Problemas de Conducta, Dificultades de Hiperactividad, Problemas con Compañeros y Conducta Prosocial. Las primeras cuatro subescalas forman una puntuación Total de Dificultades. A mayor puntuación mayor nivel de dificultades emocionales y comportamentales, excepto para la subescala Conducta Prosocial, donde una menor puntuación se corresponde con un peor ajuste en el comportamiento. Ha sido utilizado como herramienta de cribado y análisis epidemiológico del estado de salud mental en población infantojuvenil y sus propiedades psicométricas se encuentras ampliamente respaldadas (FonsecaPedrero, Paino, Lemos-Girádez, \& Muñiz, 2011; Ortuño-Sierra, Fonseca-Pedrero, Paino, \& Aritio-Solana, 2014). En este trabajo la estimación de la fiabilidad de las puntuaciones para la puntuación total de dificultades del SDQ fue de 0.81 .

La escala Rasgo de Metaconocimiento sobre Estados Emocionales-24 (Trait Meta-
Mood Scale-24, TMMS-24) (Salguero et al., 2010). La escala Rasgo de Metaconocimiento sobre Estados Emocionales-24 es un autoinforme compuesto por 24 ítems y diseñado para la valoración de los aspectos reflexivos de la experiencia emocional. El formato de respuesta es Likert de 5 puntos $(1=$ Completamente en desacuerdo y $5=$ Completamente de acuerdo) distribuidos en tres dimensiones (8 ítems cada una): Atención (conciencia) a los propios sentimientos, Claridad emocional y Reparación de las propias emociones. Estudios previos indican que es un instrumento de medida con un adecuado comportamiento psicométrico referido tanto a la consistencia interna como a la fiabilidad test-retest y a la acumulación de diferentes evidencias de validez. En este estudio, se ha utilizado la versión del TMMS-24 validada en población española (FernándezBerrocal, Extremera-Pacheco, \& Ramos, 2004). En este trabajo la estimación de la fiabilidad de las puntuaciones para cada una de las subescalas del TMMS-24 osciló entre 0.72 y 0.85 .

Escala de Autoestima de Rosenberg (Rosenberg, 1965). Es un instrumento que se basa en el modelo unidimensional de Guttman, su función específica es medir la autoestima global. El planteamiento en función del cual se construye esta escala está basado en cuatro criterios: Facilidad de administración, Economía de tiempo, Unidimensionalidad y Validez aparente. Consta de 10 ítems, de los que cinco son expresados en positivo y cinco en negativo. Su distribución es al azar para evitar aquiescencia. Este instrumento es una escala unidimensional compuesta por 10 ítems (por ejemplo, "En general estoy satisfecho conmigo mismo/a") que deben ser puntuados en una escala Likert de 5 puntos $(1=$ Totalmente en desacuerdo y $4=$ Totalmente de acuerdo). En el presente trabajo se ha utilizado la versión española cuyas propiedades psicométricas han sido analizadas en estudios previos (Rosenberg \& Owens, 2001). En este trabajo la estimación de la fiabilidad de las puntuaciones fue superior a 0.70 .

Índice de Reactividad Interpersonal (IRI) (Davis, 1980). El IRI es un cuestionario tipo autoinforme que mide las diferencias individuales 
percibidas en empatía disposicional. Consta de 28 ítems en una escala de respuesta Likert de 5 puntos $(0=$ No me describe bien $\mathrm{y}$ $4=$ Me describe muy bien). Los ítems se encuentran distribuidos en cuatro subescalas que miden cuatro dimensiones del concepto global de empatía: Toma de perspectiva, Fantasía, Preocupación empática y Malestar personal. En el presente trabajo se ha utilizado la versión española del IRI que presenta adecuadas propiedades psicométricas (Pérez-Albéniz, De Paúl, Etxeberría, Montes, \& Torres, 2003). En este trabajo la estimación de la fiabilidad de las puntuaciones para cada una de las subescalas del IRI fue superior a $0, .70$.

Cuestionario de satisfacción con el programa de Educación emocional. Se desarrolló un cuestionario para analizar la satisfacción del programa de los alumnos y de los padres del grupo experimental. Para la construcción del cuestionario se siguieron las directrices internacionales (American Educational Research Association, American Psychological Association, \& National Council on Measurement in Education 2014; Muñiz \& Fonseca-Pedrero, 2019). El cuestionario de satisfacción dirigido a los participantes del estudio consta de 33 ítems. Las primeras 20 preguntas tratan sobre la satisfacción de los participantes con diferentes aspectos del programa. Las diez siguientes preguntas valoran la satisfacción con cada una de las sesiones presentadas. Todas ellas se evalúan con una escala Likert de 5 puntos $(1=$ Totalmente de acuerdo y $5=$ Totalmente en desacuerdo). Asimismo, el cuestionario incluye una pregunta sobre la valoración global del programa con una escala Likert de 10 puntos (siendo diez la valoración más alta del programa). Adicionalmente, se pregunta a los participantes sobre si repetirían el curso y si recomendarían o no el programa y se solicita finalmente, mediante una pregunta abierta, aspectos de mejora del programa.

\section{Procedimiento}

Este estudio se enmarca dentro del plan regional de Salud Mental Riojano 2016-20. La investigación cuenta con el respaldo de las Consejerías de Salud y Educación, Formación y Empleo del Gobierno de La Rioja. El contacto con el centro escolar en el que se llevó a cabo se realizó directamente con el equipo directivo.

La administración de los cuestionarios en las evaluaciones pre y post se realizó de forma colectiva en grupos de entre 10 y 30 participantes, antes y después de la intervención. La aplicación del programa de educación emocional se realizó en un total de 10 sesiones por dos alumnos del Máster en Intervención e Innovación Educativa de la Universidad de La Rioja.

El programa adaptado para adolescentes se llevó a cabo a través de ocho sesiones todos los lunes por la mañana en un IES de La Rioja, en dos grupos aula del segundo curso de la ESO.

Se informó en todo momento de la confidencialidad de las respuestas, así como del carácter voluntario de la participación y no se dio gratificación alguna por su colaboración en el estudio. Siempre que fue necesario se solicitó un consentimiento que autorizase la participación del adolescente en la investigación.

Programa de Intervención. El programa de intervención desarrollado se fundamenta en el programa INTEMO $(+)$, utilizado previamente en adolescentes (Cabello et al., 2016) aunque con algunas modificaciones, es por ello que se le renombró como INTEMO-UR. Es un programa en el que se adaptan los ejemplos y las actividades a la edad del alumnado para que pueda utilizarse en adolescentes de 14-16 años (Extremera-Pacheco \& FernándezBerrocal, 2013). La organización de las sesiones, es en cuatro fases, que correspondió a las cuatro ramas del modelo de IE propuesto por los autores Mayer y Salovey (1997). 
Análisis de datos

Se realizaron diferentes análisis de datos. En primer lugar, se calcularon los estadísticos descriptivos para las subescalas y la puntuación total de los diferentes instrumentos de medida utilizados. En segundo lugar, se compararon las puntuaciones medias en función del grupo (experimental y de comparación) y el tiempo (pre-test y post-test). Para ello se llevó a cabo un Análisis Multivariado de la Varianza (MANOVA) de medidas repetidas, tomando como factor intersujetos el grupo y el tiempo como factor intrasujeto y como variables dependientes las subescalas y la puntuación total de los instrumentos de medida utilizados. Se utilizó el valor $\lambda$ de Wilks para observar si existían diferencias estadísticamente significativas entre todas las variables dependientes tomadas en conjunto. Como índice de tamaño del efecto se empleó el estadístico eta cuadrado parcial $\left(\eta^{2}\right.$ parcial). Finalmente, se calcularon los estadísticos descriptivos para la medida de satisfacción con el programa. Para el análisis de los datos se utilizó el programa estadístico SPSS 21.0 (IBM, 2013).

\section{Resultados}

\section{Implementación del programa INTEMO-UR}

La implementación del programa de intervención en educación emocional llevado a cabo se extendió a lo largo de 10 semanas con una duración aproximada por sesión de 60 minutos. En cuanto a las actividades, como se puede observar en la Tabla 1, se encuentran distribuidas en el mismo formato que el programa original haciendo referencia a las cuatro ramas del modelo de IE. En la misma tabla se presenta, de forma abreviada, información relevante sobre las sesiones llevadas a cabo en el programa, como son los objetivos, contenidos y las modificaciones.
Tabla 1

Programa INTEMO-UR. Sesiones, objetivo fase, contenidos y modificaciones

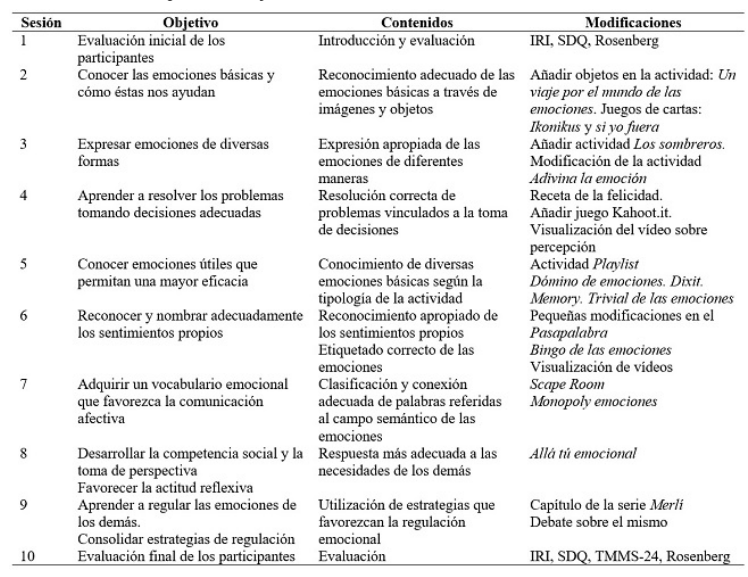

El programa INTEMO-UR se distribuyó de la siguiente manera: se establecieron diez sesiones: una sesión introductoria, ocho sesiones de actividades y una de evaluación final. La metodología utilizada fue lúdica, atractiva, vivencial, práctica, grupal, dinámica y participativa para los adolescentes, buscando sus centros de interés, donde mediante diferentes dinámicas y juegos descubrieron el fascinante mundo de las emociones. Asimismo, se trabajó también con conocimientos teóricos y conceptuales, siempre relacionados con su aplicación práctica. Se trabajaron tanto aspectos teóricos como actitudinales y de habilidad.

Efectos del entrenamiento en inteligencia emocional en indicadores de salud mental

\section{Problemas emocionales y comportamentales}

Se realizó un MANOVA considerando los dos grupos y las puntuaciones del SDQ. El valor $\lambda$ de Wilks reveló que ni el efecto del factor tiempo ( $\lambda$ Wilks $=0.99, F=0.152, p=0.69, \eta^{2}$ parcial $=0.001)$ ni su interacción con el grupo ( $\lambda$ Wilks $=0.98, F=1.46, p=0.22, \eta^{2}$ parcial $=0.014$ ) eran estadísticamente significativos. Tampoco se observó una interacción estadísticamente significativa entre los factores tiempo, grupo y dimensiones del SDQ ( $\lambda$ Wilks $=0.95, F=$ 1.07, $p=0.37, \eta^{2}$ parcial $=0.042$ ). Aunque 
no se hallaron resultados estadísticamente significativos, es digno de mención que se observó cierta mejoría en la dimensión de Problemas emocionales del SDQ (véase Figura 1 y Tabla 2).

\section{Figura 1}

Problemas emocionales en el SDQ en los grupos control y experimental y en los dos momentos temporales

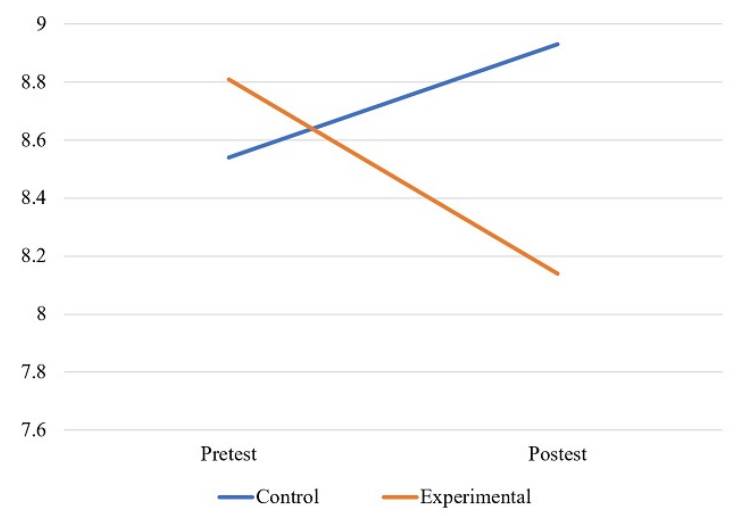

Tabla 2

Estadísticos descriptivos para todas las puntuaciones del SDQ en los dos momentos temporales y en los dos grupos

\begin{tabular}{lcccc}
\hline & \multicolumn{4}{c}{ Grupo } \\
\cline { 2 - 5 } \multicolumn{1}{c}{ Dimensiones del SDQ } & \multicolumn{2}{c}{$\begin{array}{c}\text { Control } \\
(\boldsymbol{n}=\mathbf{4 4})\end{array}$} & \multicolumn{2}{c}{$\begin{array}{c}\text { Experimental } \\
(\boldsymbol{n}=\mathbf{6 0})\end{array}$} \\
\cline { 2 - 5 } & \multicolumn{1}{c}{ Pre } & Post & Pre & Post \\
& $\mathrm{M}(D E)$ & $\mathrm{M}(D E)$ & $\mathrm{M}(D E)$ & $\mathrm{M}(D E)$ \\
\hline Problemas emocionales & $8.54(2.6)$ & $8.93(2.2)$ & $8.81(2.3)$ & $8.14(2.7)$ \\
Problemas conductuales & $7.61(1.7)$ & $7.90(2.1)$ & $7.63(1.7)$ & $7.95(2.5)$ \\
Problemas con compañeros & $6.68(1.9)$ & $7.25(2.3)$ & $6.85(2.1)$ & $6.73(2.1)$ \\
Hiperactividad & $9.93(2.1)$ & $10.27(2.2)$ & $9.93(2.5)$ & $9.85(2.7)$ \\
Prosocial & $12.63(1.6)$ & $12.18(1.9)$ & $13.30(1.6)$ & $13(2.3)$ \\
\hline
\end{tabular}

\section{Autoestima}

Para las puntuaciones de autoestima, el MANOVA reveló que ni el factor tiempo $(\lambda$ Wilks $=0.99, \mathrm{~F}=0.75, \mathrm{p}>0.07, \eta^{2}$ parcial $=$ 0.002) ni su interacción con el grupo ( $\lambda$ Wilks $=$ $0.07, F=3.45, p=0.07, \eta^{2}$ parcial $=0.070$ ) eran estadísticamente significativos. En la Tabla 3 se presentan los estadísticos descriptivos para la puntuación total de Autoestima para los dos grupos y en los momentos temporales.
Tabla 3

Estadísticos descriptivos para las puntuaciones de Autoestima en los dos momentos temporales y en los dos grupos

\begin{tabular}{ccccc}
\hline \multirow{2}{*}{ Variable } & \multicolumn{3}{c}{ Grupo } \\
\cline { 2 - 5 } & \multicolumn{2}{c}{$\begin{array}{c}\text { Control } \\
(\boldsymbol{n}=\mathbf{4 4})\end{array}$} & \multicolumn{2}{c}{$\begin{array}{c}\text { Experimental } \\
(\boldsymbol{n}=\mathbf{6 0 )}\end{array}$} \\
\hline \multirow{3}{*}{ Autoestima } & Pre & Post & Pre & Post \\
& $M(D T)$ & $M(D T)$ & $M(D T)$ & $M(D T)$ \\
\cline { 2 - 5 } & $31.59(5.5)$ & $29.81(5.2)$ & $30.86(7.0)$ & $31.68(6.8)$ \\
\hline
\end{tabular}

\section{Inteligencia emocional percibida}

Se realizó un MANOVA considerando los dos grupos y las puntuaciones del TMMS-24. Los resultados no revelaron diferencias estadísticamente significativas para el efecto principal del grupo $\left(F=3.046, p=0.090, \eta^{2}\right.$ parcial $=0.082)$. Del mismo modo, no se hallaron diferencias estadísticamente significativas para el factor del tiempo ( $\lambda$ Wilks $=0.98, F=0.69, p$ $=0.41, \eta^{2}$ parcial $\left.=0.02\right)$, ni para su interacción con el grupo $(\lambda$ Wilks $=0.98, F=0.37, p=0.54$, $\eta^{2}$ parcial $\left.=0.01\right)$. En la Tabla 4 se presentan los estadísticos descriptivos para la puntuación total de TMMS-24 y sus subescalas en los dos momentos temporales.

\section{Tabla 4}

Estadísticos descriptivos para todas las puntuaciones del TMMS-24 en los dos momentos temporales y en los dos grupos

\begin{tabular}{lcccc}
\hline & \multicolumn{4}{c}{ Grupo } \\
\cline { 2 - 5 } \multicolumn{1}{c}{$\begin{array}{c}\text { Dimensiones } \\
\text { TMMS-24 }\end{array}$} & \multicolumn{2}{c}{$\begin{array}{c}\text { Control } \\
(\boldsymbol{n}=\mathbf{4 4})\end{array}$} & \multicolumn{2}{c}{$\begin{array}{c}\text { Experimental } \\
(\boldsymbol{n}=\mathbf{6 0})\end{array}$} \\
\cline { 2 - 5 } & $\begin{array}{c}\text { Pre } \\
\text { M }(\boldsymbol{D} \boldsymbol{T})\end{array}$ & $\begin{array}{c}\text { Post }(\boldsymbol{D} \boldsymbol{T}) \\
\text { Pre }\end{array}$ & $\begin{array}{c}\text { Post }(\boldsymbol{D} \boldsymbol{T}) \\
\mathbf{M}(\boldsymbol{D} \boldsymbol{T})\end{array}$ \\
\hline Percepción & $25.11(5.9)$ & $25.25(4.8)$ & $27.20(5.6)$ & $27.73(6.6)$ \\
Comprensión & $26.79(5.9)$ & $26.09(5.1)$ & $26.75(5.4)$ & $28.93(5.7)$ \\
Regulación & $28.61(5.9)$ & $27.59(4.9)$ & $27.73(6.6)$ & $28.01(7.1)$ \\
\hline
\end{tabular}

\section{Empatía}

Se realizó un MANOVA considerando los dos grupos y las puntuaciones del IRI. Los resultados no revelaron un efecto principal del grupo estadísticamente significativo $(F=3.046, p=$ 0.090, $\eta^{2}$ parcial $=0.082$ ). Del mismo modo, ni el efecto del tiempo ( $\lambda$ Wilks $=0.99, F$ $=0.59, p=0.80, \eta^{2}$ parcial $\left.=0.01\right)$, ni su 
interacción con el grupo fueron significativos $(\lambda$ Wilks $=0.97, F=0.72, p=0.54, \eta^{2}$ parcial $=$ 0.21). En la Tabla 5 se presentan los estadísticos descriptivos para las dimensiones del IRI en los dos momentos temporales. Las puntuaciones medias fueron elevadas.

\section{Tabla 5}

Estadísticos descriptivos para todas las puntuaciones del IRI en los dos momentos temporales y en los dos grupos

\begin{tabular}{lcccc}
\hline & \multicolumn{4}{c}{ Grupo } \\
\cline { 2 - 5 } \multicolumn{1}{c}{ Dimensiones IRI } & \multicolumn{2}{c}{$\begin{array}{c}\text { Control } \\
(\boldsymbol{n}=\mathbf{4 4})\end{array}$} & \multicolumn{2}{c}{$\begin{array}{c}\text { Experimental } \\
(\boldsymbol{n}=\mathbf{6 0})\end{array}$} \\
\cline { 2 - 5 } & \multicolumn{1}{c}{$\begin{array}{c}\text { Pre } \\
\boldsymbol{M}(\boldsymbol{D} \boldsymbol{T})\end{array}$} & $\boldsymbol{M}(\boldsymbol{D} \boldsymbol{T})$ & $\boldsymbol{M}(\boldsymbol{D} \boldsymbol{T})$ & $\boldsymbol{M}(\boldsymbol{D} \boldsymbol{T})$ \\
\hline Toma de perspectiva & $22.13(4.0)$ & $22.00(3.1)$ & $23.26(4.5)$ & $23.70(4.4)$ \\
Fantasía & $22(4.5)$ & $21.38(4.5)$ & $22.96(5.5)$ & $24.36(5.3)$ \\
Preocupación empática & $26.34(3.8)$ & $25.68(3.3)$ & $27.66(3.6)$ & $27.91(4.0)$ \\
Malestar personal & $16.56(4.8)$ & $16.61(3.2)$ & $17.31(3.8)$ & $17.28(4.1)$ \\
\hline
\end{tabular}

Satisfacción de los participantes

A continuación, se examinó el grado de satisfacción con el programa de educación emocional implementado. Los resultados referidos a los estadísticos descriptivos de las puntuaciones se recogen en la Tabla 6. Los ítems que presentaron mayor puntuación media fueron los que hacen referencia al trato recibido de los profesores, a la corta duración del programa y la recreación de las dinámicas. Los ítems que presentaron menor puntuación fueron los referentes a las evaluaciones inicial y final donde se les administraron los test. Las dinámicas que mejor valoración obtuvieron fueron la sesión 7 y la sesión 9. Es digno de mención que el $100 \%$ de los alumnos volvería a repetir este curso y además recomendarían este programa. La valoración general de los participantes del programa mostró que el 100\% de los alumnos puntuó por encima de 7 puntos. La puntuación media de satisfacción del programa por parte de los alumnos participantes fue de $8.9(D T=0.85)$.

\section{Tabla 6}

Estadísticos descriptivos para los ítems de la encuesta de satisfacción

\begin{tabular}{|c|c|c|c|c|}
\hline İtem & $M$ & $D T$ & Min. & Max: \\
\hline Los contenidos han cubierto mis expectativas. & 4.2 & 0.7 & 2 & 5 \\
\hline Los contenidos me han gustado. & 4.6 & 0.6 & 3 & 5 \\
\hline Los temas se han tratado con la profundidad que esperaba. & 4.1 & 0.8 & 2 & 5 \\
\hline Los contenidos aprendidos han sido útiles para mi vida. & 4.1 & 0.7 & 2 & 5 \\
\hline Los ejercicios prácticos han sido de interés. & 4.5 & 0.7 & 3 & 5 \\
\hline Las dinámicas de grupo han sido divertidas. & 4.7 & 0.5 & 4 & 5 \\
\hline Me han gustado los materiales que se han dado. & 4.4 & 0.6 & 3 & 5 \\
\hline El aula y mobiliario han sido adecuadas. & 4.3 & 0.7 & 3 & 5 \\
\hline Los medios y materiales utilizados para las dinámicas me han gustado. & 4.4 & 0.7 & 2 & 5 \\
\hline Me ha parecido que el programa ha durado poco tiempo. & 4.6 & 0.7 & 1 & 5 \\
\hline Los profesores dominan la materia. & 4.8 & 0.5 & 3 & 5 \\
\hline Los profesores explican con claridad. & 4.7 & 0.5 & 3 & 5 \\
\hline Los profesores son simpáticos. & 4.8 & 0.4 & 3 & 5 \\
\hline Los profesores nos han tratado con respeto y cariño. & 4.8 & 0.5 & 3 & 5 \\
\hline Los profesores motivan y despiertan mi interés. & 4.4 & 0.6 & 3 & 5 \\
\hline Creo que el programa ha mejorado mis recursos emocionales. & 4 & 0.8 & 2 & 5 \\
\hline El programa me ha ayudado a mejorar mis relaciones con los demás. & 3.8 & 0.9 & 1 & 5 \\
\hline Desde que vengo al programa conozco mejor lo que soy capaz de hacer. & 3.9 & 0.9 & 2 & 5 \\
\hline Creo que se deberia impartir este programa desde el colegio/instituto. & 4.5 & 0.7 & 2 & 5 \\
\hline Estoy contento con mi participación en el programa. & 4.4 & 0.7 & 3 & 5 \\
\hline Sesión N. ${ }^{\circ}$ (Test) & 2.8 & 1.4 & 1 & 5 \\
\hline Sesión N.22 (Objetos sobre emociones) & 4 & 0.8 & 2 & 5 \\
\hline Sesión N.?3 (Música emocional y el juego-sombreros) & 4.4 & 0.7 & 2 & 5 \\
\hline Sesión N.94 (Búnker, kahoot y karaoke) & 4.2 & 0.9 & 1 & 5 \\
\hline Sesión N. ${ }^{\circ 5}$ (Dominó, Memory, Trivial, Dixit) & 4.4 & 0.7 & 2 & 5 \\
\hline Sesión N.6 (Pasapalabra, Bingo) & 4.4 & 0.7 & 3 & 5 \\
\hline Sesión N. ${ }^{\circ 7}$ (Scape Room) & 4.7 & 0.6 & 3 & 5 \\
\hline Sesión N.8 (Empatía) & 4.2 & 0.8 & 2 & 5 \\
\hline Sesión N.99 (Película) & 4.6 & 0.7 & 1 & 5 \\
\hline Sesión N. ${ }^{\circ} 10$ (Post test) & 3 & 1.4 & 1 & 5 \\
\hline
\end{tabular}

\section{Discusión}

La literatura previa analizada indica que, dentro de los programas emocionales para adolescentes, existen escasas investigaciones de carácter empírico que analicen los posibles efectos beneficiosos que estas intervenciones pudieran tener a nivel de gestión y manejo de las emociones y de las relaciones sociales, así como otros aspectos de la salud mental y el bienestar emocional (Andrés-Cruz, 2012). No cabe duda que es sumamente importante diseñar programas educativos con la finalidad de desarrollar y mejorar las habilidades emocionales de los jóvenes. Es necesario, además, evaluar científicamente estas intervenciones para que aporten evidencias empíricas que validen y certifiquen dichos procesos y su grado de efectividad, así como ayudar a divisar los aspectos que sean susceptibles de mejora. En este contexto, el objetivo general del presente trabajo fue implementar y examinar el efecto de un programa de educación emocional en adolescentes de la población general.

El primer objetivo fue implementar un programa de educación emocional con la finalidad de desarrollar, promover y/o potenciar 
las competencias socio-emocionales de los adolescentes. En esta investigación, tras la implantación del programa INTEMO-UR, no se han evidenciado mejoras estadísticamente significativas en los alumnos. Sin embargo, sí se puede afirmar que el programa es novedoso y se adecua a la realidad educativa actual porque está ajustado y diseñado para adolescentes, los cuales son capaces de percibir una leve mejoría y mostrar alta satisfacción general con la nueva forma de aprender. Por lo tanto, se necesitan nuevas fórmulas, programas y formas de aprender, donde estén implícitas las emociones (Bisquerra et al., 2012). Los programas de educación emocional parece que se pueden implementar en el contexto educativo de los jóvenes de forma adecuada.

El segundo objetivo fue analizar los posibles beneficios del programa de educación emocional sobre diferentes indicadores de salud mental, como problemáticas emocionales y comportamentales, autoestima, empatía e inteligencia emocional percibida. Los resultados, en primer lugar, confirman que el programa INTEMO (con ligeras modificaciones) ha mejorado algunos aspectos relevantes de la IE, aunque no significativamente. Trabajos previos utilizando el INTEMO han demostrado su efectividad, indicando que tiene efectos positivos a corto y a largo plazo en aspectos tan relevantes como el ajuste psicológico, las relaciones sociales, la empatía o las conductas disruptivas y violentas (Castillo et al., 2013; Ruiz-Aranda, et al., 2012; Ruiz-Aranda, et al., 2011). Otros estudios realizados revelaron que los programas de IE mejoran la calidad de vida de los adolescentes tratando de optimizar sus capacidades cognitivas, emocionales y sociales (Jiménez \& López-Zafra, 2010; Jiménez, Palmero \& Luis, 2013). También algunos trabajos previos demostraron que los programas de aprendizaje socioemocional son sugestivos para disminuir y prevenir problemas psicológicos y comportamentales, así como ayudar a un mayor ajuste social y mental entre los adolescentes y prevenir múltiples problemas psicológicos (Durlak, Weissberg, Dymnicki, Taylor, \& Schellinger, 2011; Lopes, Grewal, Kadis, Gall, \& Salovey, 2006; Mayer et al., 2008).
Los programas de educación emocional pueden disminuir las conductas desadaptadas y favorecer el bienestar general en una doble vía, a un nivel más intrapersonal, dotando de estrategias para la regulación emocional y social, y a nivel interpersonal, incrementando las estrategias para manejar las emociones de los demás, (RuizArandaet al., 2011; Oliva, 2015).

En general, los hallazgos en programas denominados genéricamente como de educación emocional reflejaron que son capaces de impulsar distintas habilidades relacionadas con la salud mental en jóvenes, disminuyendo distintos factores de riesgo y trastornos de conducta (Castillo et al., 2013; Ruiz-Aranda et al., 2012; Extremera-Pacheco \& FernándezBerrocal, 2004; Fernández-Berrocal et al., 2004). A pesar de no hallar diferencias estadísticamente significativas en este trabajo, es interesante indicar que la educación en este conjunto de habilidades sirve para tomar conciencia de las propias emociones y las de las demás personas. Además, capacita para comprenderlas y regularlas, tiene efectos beneficiosos a múltiples niveles sobre la salud física y psicológica de los adolescentes y repercute en un mejor rendimiento académico (Extremera-Pacheco \& Fernández-Berrocal, 2013). No obstante, es posible que algunos programas emocionales (o cualquiera de sus componentes) diseñados para adolescentes muestren sus efectos en periodos más dilatados en el tiempo, al cabo de años y no en periodos cortos, por ejemplo, de meses, como es el caso del presente estudio. También es posible que el no encontrar resultados estadísticamente significativos sea debido al reducido tamaño muestral y/o la incapacidad de las medidas para captar con suficiente sensibilidad los cambios producidos. Sea como fuere, a través de la educación emocional es como se pretende conseguir el desarrollo integral de los jóvenes, siendo un proceso educativo continuo y permanente (Bisquerra et al., 2015).

El tercer objetivo fue analizar la satisfacción de los usuarios que han participado en el programa de educación emocional INTEMO-UR. La gran mayoría de los participantes mostraron niveles elevados de satisfacción hacia el programa de 
educación emocional INTEMO-UR, siendo los comentarios más frecuentes aspectos relativos a la falta de tiempo, actividades en espacios que obliguen a salir del aula y actividades que ayuden a situarte en el lugar de los otros. Los resultados de este estudio indican que todos los participantes de la intervención recomendarían el programa y volverían a repetirlo. Estos resultados son reveladores y manifiestan una alta satisfacción por parte de los participantes con el programa, con los educadores y con las sesiones.

Existen ciertas limitaciones en relación a este estudio y a los resultados obtenidos. En primer lugar, el reducido tamaño muestral que se utilizó para la presente investigación, así como la escasa diversidad de centros educativos puede afectar a los resultados encontrados. No obstante, es digno de mención que el tipo de diseño cuasiexperimental y el programa de intervención empleado (10 sesiones en un periodo de 3 meses) son fortalezas de la presente investigación. En segundo lugar, y como se comentó, hubiese sido interesante realizar seguimiento longitudinal a 6 y 12 meses para comprobar los efectos del programa de intervención. En tercer lugar, hubiese sido interesante recoger información de otras fuentes e informantes como padres o profesores y no sólo mediante autoinformes. Por ello, en futuras investigaciones no cabe duda que sería muy recomendable utilizar otras medidas de evaluación de la eficacia del programa, como, por ejemplo, evaluación del funcionamiento neurocognitivo (e.g., atención, memoria, cognición social, funciones ejecutivas, etc.) o la satisfacción de los profesores o de las familias involucradas en el programa. Además, sería interesante implementar nuevos modelos psicométricos como el análisis de redes (FonsecaPedrero, 2018), nuevas formas de evaluación, como la evaluación ambulatoria (Myin-Germeys et al., 2018) o un programa de intervención con un periodo temporal más dilatado en el tiempo.

\section{Agradecimientos}

Esta investigación ha sido financiada por la Convocatoria 2015 de Ayudas Fundación BBVA a Investigadores y Creadores Culturales, por las Ayudas Fundación BBVA a Equipos de Investigación Científica 2017, por el Instituto de Estudios Riojanos (IER) del Gobierno de La Rioja, y por cofinanciado con fondos FEDER en el PO FEDER de La Rioja 2014-2020 (SRS 6FRSABC026).

\section{Referencias}

American Educational Research Association, American Psychological Association, \& National Council on Measurement in Education. (2014). Standards for educational and psychological testing. Washington, DC: American Psychological Association.

Andrés-Cruz, V. (2012). Inteligencia emocional y bienestar subjetivo: un estudio en adolescentes. Fòrum de Recerca, 17, 229-242. http://dx.doi.org/10.6035/Forum Recerca.2012.17.14

Bisquerra, R. (2011). Educación emocional: Propuesta para educadores y familiares. Bilbao: Desclée de Brouwer.

Bisquerra, R., Punset, E., Mora, F., Garcia, E., Lopez, E., Perez, J., ... Planells, O. (2012). ¿Cómo educar las emociones? La inteligencia emocional en la infancia y la adolescencia. Barcelona, España: Hospital Sant Joan de Déu.

Bisquerra, R., Pérez-González. J. C., \& García, E. (2015). Inteligencia emocional en educación. Madrid: Síntesis.

Bousoño, M., Al-Halabí, S., Burón, P., Garrido, M., Díaz-Mesa, E., Galván, G, ... Bobes, J. (2017). Uso y abuso de sustancias psicotrópicas e internet psicopatología e ideación suicida en adolescentes. Adicciones, 29(2), 97-104. https://doi.org/1 0.20882 /adicciones. 811

Carli, V., Hoven, C.W., Wasserman, C., Chiesa, F., Guffanti, G., Sarchiapone, ... Wasserman, D., (2014). A newly identified group of adolescents at "invisible" risk for psychopathology and suicidal behavior: findings from the SEYLE study. World 
Psychiatry, 13(1), 78-86. https://doi.org/10. 1002/wps.20088

Cabello, R., Castillo, R., Rueda, P., \& FernándezBerrocal, P. (2016). Programa INTEMO +. Mejorar la inteligencia emocional de los adolescentes. Madrid: Pirámide.

Castillo, R., Salguero, J. M., FernándezBerrocal, P., \& Balluerka, N. (2013). Effects of an emotional intelligence intervention on aggression and empathy among adolescents. Journal of Adolescence, 36(5), 883-892. https://doi.org/10.1016/j.a dolescence.2013.07.001

Catalá-López, F., Gènova-Maleras, R., ÁlvarezMartín, E., de Larrea-Baz, N. F., \& MorantGinestar, C. (2013). Carga de enfermedad en adolescentes y jóvenes en España. Revista de Psiquiatría y Salud Mental, 6(2), 80-85. https://doi.org/10.1016/j.rpsm.2012. 07.002

Davis, M. H. (1980). A multidimensional approach to individual differences in empahty. Catalog of Selected Document in Psychology, 10, 1-85. Recuperado de https:/ /www.uv.es/ friasnav/Davis_1980.pdf

Durlak, J. A., Weissberg, R. P., Dymnicki, A. B., Taylor, R. D., \& Schellinger, K. B. (2011). The impact of enhancing students' social and emotional learning: A meta-analysis of school-based universal interventions. Child Development, 82 (1), 474-501. https://doi.or g/10.1111/j.1467-8624.2010.01564.x

Extremera-Pacheco, N., \& Fernández-Berrocal, P. (2004). Inteligencia emocional, calidad de las relaciones interpersonal y empatía en estudiantes universitarios. Clínica y Salud, 15(2), 117-137. Recuperado de http://www .redalyc.org/pdf/1806/180617822001.pdf

Extremera-Pacheco, N., \& Fernández Berrocal, P. (2013). Inteligencia emocional en adolescentes. Padres y Maestros, 352, 34-39. Recuperado de http://www.web.teaediciones.com/Ejem plos/PyM_Orientacion\%20Educativa\%203 4_39.pdf

Fernández-Berrocal, P., Extremera-Pacheco, N., $\&$ Ramos, N. (2004). Validity and reliability of the Spanish modified version of the Trait
Meta-Mood Scale. Psychological Reports, 94, 751-755. https://doi.org/10.2466/pr0.94.3. 751-755

Fonseca-Pedrero, E., Paino, M., LemosGirádez, S., \& Muñiz, J. (2011). Prevalencia de la sintomatología emocional y comportamental en adolescentes españoles a través del Strengths and Difficulties Questionnaire (SDQ). Revista de Psicopatología y Psicología Clínica, 16(1), 15-25. https://doi.org/10.5944/rppc.vol.16. num.1.2011.10348

Fonseca-Pedrero, E. (2018). Análisis de redes en psicología. Papeles del Psicólogo, 39(1), 1-12. https://doi.org/10.23923/pap.psicol20 18.2852

Goodman, R. (1997). The strengths and difficuties questionnaire: a research note. Journal of Child Psychology and Psychiatry, 38(5), 581-586. https://doi.org/10.1111/j.1 469-7610.1997.tb01545.x

IBM. (2013). IBM. SPSS. Stadistics for Windows, versión 21.0 Armonk. NY: IBM Corp.

Jiménez, M. I., \& López-Zafra, E. (2010). Inteligencia emocional percibida $y$ rendimiento académico en estudiantes españoles de enseñanza secundaria obligatoria. En J. J. Gázquez \& Mạ. C. Pérez (Coords.), Investigación en convivencia escolar: Variables relacionadas (pp. 505-508). Almería: Geu.

Jiménez, A., Palmero, C., \& Luis, I. (2013). Gestión de la calidad en los programas universitarios de personas mayores: Implicaciones para el alumnado. International Journal of Developmental and Educational Psychology, 2(1), 347-354. Recuperado de http://dehesa.unex.es/bitstream/handle/ 10662/5674/0214-9877_2013_1_2_347.pd $\mathrm{f}$ ? sequence $=1$ \&isAllowed $=\mathrm{y}$

Lopes, P. N., Grewal, D., Kadis, J., Gall, M. \& Salovey, P. (2006). Evidence that emotional intelligence is related to job performance and affect and attitudes at work. Psicothema, 18(supl.), 132-138.

Mayer, J. D., Caruso, D. R., \& Salovey, P. (2000). Selecting a measure of emotional 
intelligence: The case of ability scales. En R. Bar-On \& J. D. A. Parker (Eds.), The handbook of emotional intelligence: Theory, development, assessment and application at home, school, and in the workplace (pp. 320-342). San Francisco: Jossey-Bass.

Mayer, J. D., Roberts, R. D., \& Barsade, S. G. (2008). Human abilities: Emotional intelligence. Annual Review of Psychology, 59, 507-536. https://doi.org/10.1146/annur ev.psych.59.103006.093646

Mayer, J. D., \& Salovey, P. (1997). What is emotional intelligence? En P. Salovey \& D. J. Sluyter (Eds.), Emotional development and emotional intelligence: Educational implications (pp. 3-31). New York, NY: Basic Books.

Merikangas, K. R., He, J., Burstein, M., Swanson, S. A., Avenoli, S., Cui, ... Swendsen, J. (2010). Lifetime Prevalence of mental disorders in U.S. adolescents: results from the National Comorbidity Survey Replication-Adolescents Spplement (NCSA). Journal of the American Academy of Child and Adolescent Psychiatry, 49, 980-989.

Mestre-Navas, J. M., \& Fernández-Berrocal, P. (2007). Manual de inteligencia emocional. Madrid: Pirámide.

Muñiz, J., \& Fonseca-Pedrero, E. (2019). Diez pasos para la construcción de test. Psicothema, 31 (1), 7-16. https://doi.org/10.7 334/psicothema2018.291

Myin-Germeys, I., Kasanova, Z., Vaessen, T., Vachon, H., Kirtley, O., Viechtbauer, W., \& Reininghaus, U. (2018). Experience sampling methodology in mental health research: New insights and technical developments. World Psychiatry, 17(2), 123-132. https://doi.org/10.1002/wps.2051 3

Oliva, A. (2015). Desarrollo Positivo Adolescente. Madrid: Síntesis.

Ortuno-Sierra, J., Fonseca-Pedrero, E., Paino, M., \& Aritio-Solana, R. (2014). Prevalence of emotional and behavioral symptomatology in Spanish adolescents. Revista de Psiquiatría y Salud Mental, 7(3),
121-130. https://doi.org/10.1016/j.rpsm.20 13.12 .003

Parés-Badell, O., Barbaglia, G., Jerinic, P., Gustavsson, A., Salvador-Carulla, L., \& Alonso, J. (2014). Cost of disorders of the brain in Spain. PLoS ONE, 9(8), 1-10. http s://doi.org/10.1371/journal.pone.0105471

Pérez-Albéniz, A., De Paúl, J., Etxeberría, J., Montes, M. P., \& Torres, E. (2003). Adaptación de Interpersonal Reactivity Index (IRI) al español. Psicothema, 15(2), 267-272. Recuperado de http://www.psicot hema.com/psicothema.asp? $\mathrm{id}=1056$

Pérez-González, J. L. (2008). Propuesta para la evaluación de programas socioemocionales. Revista Electrónica de Investigación Psicoeducativa, 6(2), 523-546. Recuperado de http://www.investigacion-psicopedagogi ca.org/revista/articulos/15/espannol/Art_1 5 246.pdf

Repetto-Talavera, E., \& Pérez-González, J. (2007). formación en competencias socioemocionales a través de las prácticas en empresas. Revista Europea de Formación Profesional, 40, 92-112. Recuperado de https://www.researchgate.net/publicatio n/39221596_Formacion_en_competencias _socioemocionales_a_traves_de_las_practi cas_en_empresas

Rosenberg, M. (1965). Society and the adolescent self-image. Princeton: Princeton University Press. https://doi.org/10.1515/9781400876 136

Rosenberg, M., \& Owens, T. J. (2001). Low selfesteem people: A collective portrait. En T. J. Owens, S. Stryker \& N. Goodman (Eds.), Exending self-esteem theory and research: Sociological and psychological currents (pp. 400-436). New York, NY: Cambridge University Press. https://doi.org/10.1017/C BO9780511527739.018

Ruiz-Aranda, D., Cabello, R., Salguero, J. M., Palomera, R., Extremera, N., \& FernándezBerrocal, P. (2013). Guía para mejorar la inteligencia emocional de los adolescentes. Programa INTEMO. Madrid: Pirámide.

Ruiz-Aranda, D., Castillo, R., Salguero, J. M., Cabello, R., Fernández-Berrocal, P., \& 
Balluerka, N. (2012). Short- and midterm effects of emotional intelligence training on adolescent mental health. Journal of Adolescent Health, 51(5), 462-467. https://d oi.org/10.1016/j.jadohealth.2012.02.003

Ruiz-Aranda, D., Salguero, J. M., Cabello, R., Palomera, R., \& FernándezBerrocal, P. (2011). Can an emotional intelligence program improve adolescents' psychosocial adjustment? Social Behavior and Personality an International Journal, 40 (8), 1373-1380. https://doi.org/10.2224/s bp.2012.40.8.1373

Salguero, J. M., Fernandez-Berrocal, F., Balluerka, N., \& Aritzeta, A. (2010). Measuring perceived emotional intelligence in the adolescent population: psychometric properties of the trait metamood scale. Social Behavior and Personality, 38(9), 1197-1210. https://doi.org/10.2224/s bp.2010.38.9.1197

Sánchez, M. A., Pérez, A., Paino, M., \& FonsecaPedrero, E. (2018). Ajuste Emocional y Comportamental en una Muestra de Adolescentes Españoles. Actas Españolas de Psiquiatría, 46(6), 205-216. Recuperado de https://medes.com/publication/141275

\section{Notas}

* Artículo de investigación. 